\title{
Do we need three coronary arteries?
}

\author{
Santosh Sinha* \\ Department of Cardiology, LPS Institute of Cardiology, G.S.V.M. Medical College, India
}

\begin{abstract}
Normal myocardium is usually perfused by three coronary arteries- a left main system bifurcating into left anterior descending artery (LAD), and left circumflex artery $(\mathrm{LCx})$, and right coronary artery (RCA). Coronary artery anomalies are congenital changes in their origin, course, and/or structure, which are usually discovered as incidental findings during coronary angiographic studies. Here, we report a case of 56-year-old hypertensive, and diabetic male who was being evaluated for exertional angina and dyspnoea whose coronary angiogram revealed a rare coronary anomaly: normal LAD, absent LCx, and superdominant RCA which was better delineated on Multidetector coronary angiography (MDCT). Multislice CT is the non-invasive modality of choice for imaging anomalies of coronary arteries and provides high speed with excellent spatial resolution.
\end{abstract}

\section{Introduction}

Coronary artery anomalies (CAA) are a diverse group of congenital disorders, whose manifestations and pathophysiological mechanisms are highly variable, with a global incidence of $5.64 \%$ [1]. The classification of coronary anomalies is based on the abnormalities seen in one or more site of origin, course, termination and branches of the major coronary arteries. The absence of the left circumflex (LCX) artery with a superdominant right coronary artery (RCA) is very rare.

\section{Case report}

A 56-year old female with diabetes, and hypertension for past six years, was evaluated for exertional angina, and dyspnoea. Initially his blood pressure was 168/100 $\mathrm{mm}$ Hg prior to treatment. Blood pressure on presentation was $134 / 84 \mathrm{~mm} \mathrm{Hg}$. in the left arm in supine position with current antihypertensive drugs. Other physical examinations were unremarkable. Electrocardiogram showed left ventricular hypertrophy with strain pattern. Echocardiography revealed mild concentric left ventricular hypertrophy, grade-I diastolic dysfunction and normal ejection fraction. Exercise stress testing was inconclusive as it was prematurely terminated because of dyspnoea. Patient underwent coronary angiogram after proper consent. Cannulation of left main coronary artery showed its normal course which was continuing into left anterior descending artery (LAD) without giving left circumflex artery (LCx) (Figure 1). However, right coronary artery (RCA) showed a superdominant pattern, with various posterior descending branches, extending beyond the crux cordis and circling the atrioventricular groove almost completely, following the expected path of the absent LCx (Figure 2). Moreover, nonselective injection of contrast media into the aortic sinus ruled out its ectopic origin from either sinuses or right coronary artery itself. Both the arteries appeared free of disease. To delineate the course of artery, 128-slice multi-detector computed tomography (MDCT) coronary angiography was performed which normal LAD, absent LCx, and a superdominant RCA (Figure 3). Therefore, left main coronary artery was functionally continuing as LAD, which meant myocardium was being perfused by functionally only two coronary arteries- LAD, and RCA (Figure 3 ). He was managed conservatively with antihypertensive drugs (amlodipine- $5 \mathrm{mg}$ once daily, ramipril-5 mg once daily, and hydrochlorthiazide-12.5 mg once daily), and metformin-500 mg twice daily.

\section{Discussion}

LCx artery is the dominance vessel in 15\% of patients which gives rise to left posterior descending artery (PDA) as its distal continuation. The RCA is dominant in $85 \%$ of patients, supplying the PDA and at least one postero-lateral branch. In $7.5 \%$ of patients, left PDA and left postero-lateral branches are provided by distal LCx artery, making it dominant (left dominant). In the remaining $7.5 \%$ patients, RCA gives rise to PDA and LCx provides the entire postero-lateral branch, thus making it co-dominant circulation. A vessel is considered super-

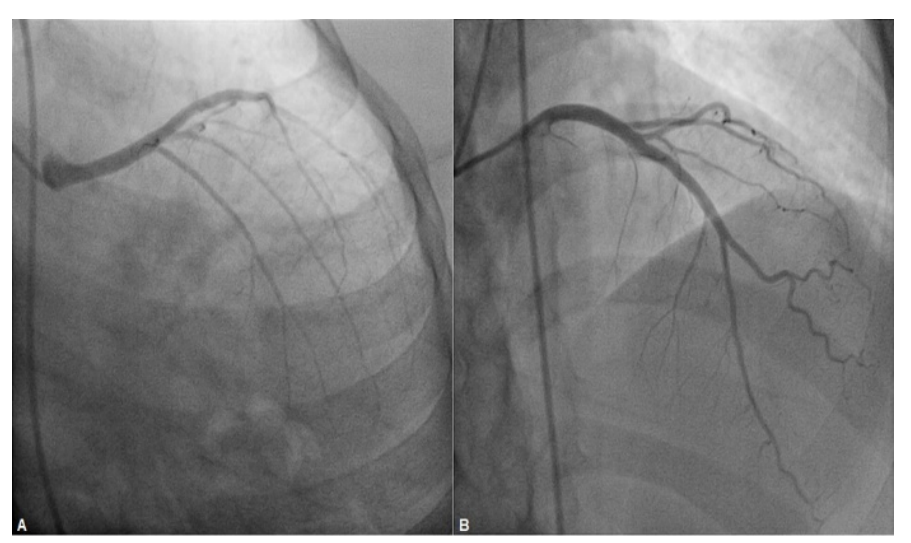

Figure 1. Coronary angiogram showing left main (LM) trunk continuing into left anterior descending artery (LAD) without giving rise to left circumflex artery (LCx) (A-anteroposterior caudal view; B-antero-posterior cranial view)

${ }^{\star}$ Correspondence to: Santosh Sinha, Department of Cardiology, LPS Institute of Cardiology, G.S.V.M. Medical College, India, E-mail: fionasan@rediffmail.com

Key words: absent left circumflex artery, coronary artery anomaly, multidetector coronary angiography, superdominant right coronary artery

Received: October 02, 2020; Accepted: October 15, 2020; Published: October 23,2020 


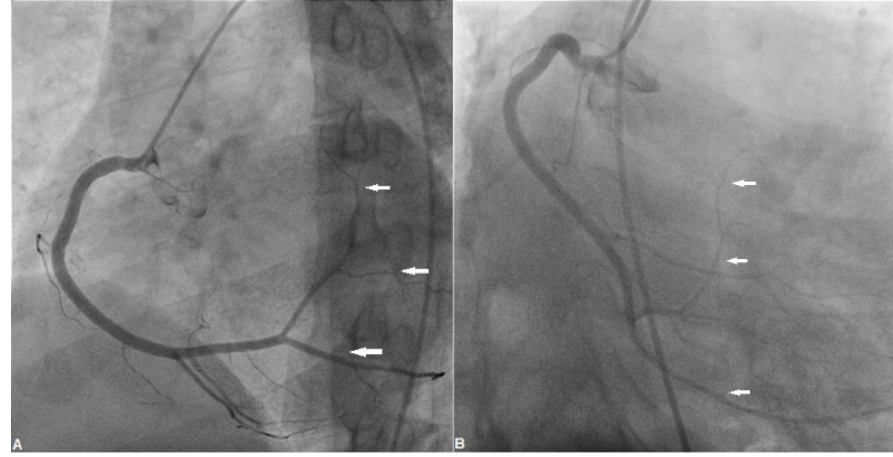

Figure 2. The superdominant right coronary artery (RCA), with various posterior descending branches (PDA), extending beyond the crux cordis and circling the atrioventricular groove, following the expected path of the absent circumflex artery (A- left anterior oblique view; B- left anterior oblique with cranial angulation)

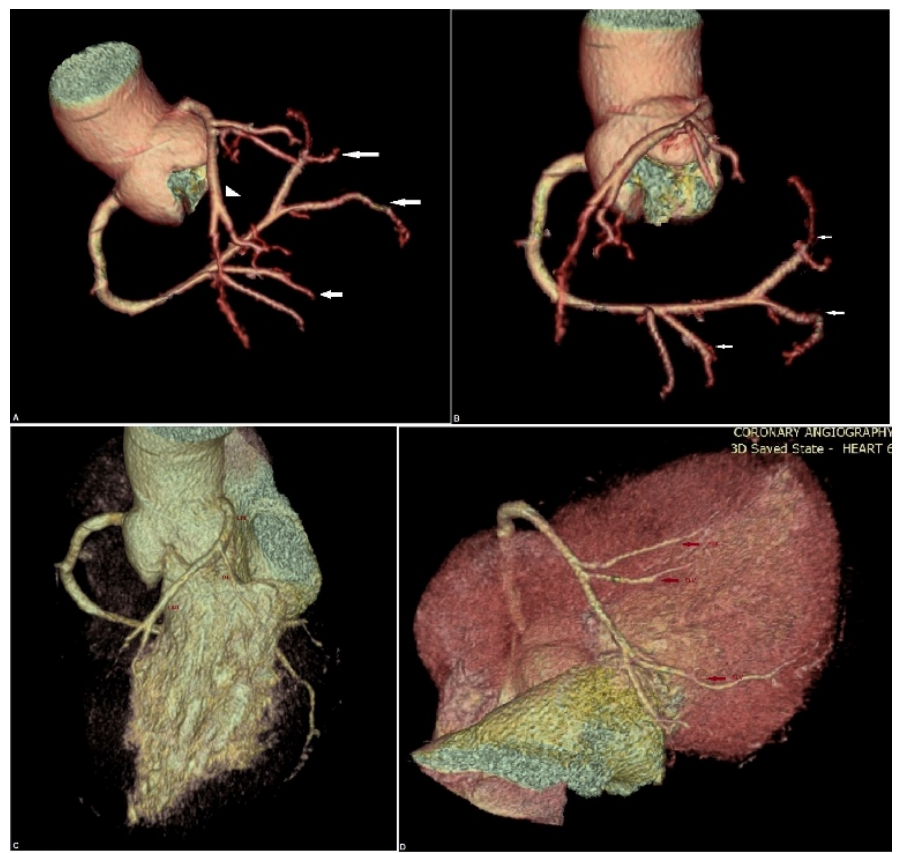

Figure 3. MDCT showing LM trunk continuing into LAD without giving rise to $\mathrm{LCx}$ and superdominant RCA, with PDA and PLV branches, extending beyond the crux cordis and circling the atrioventricular groove (A- LAO with cranial angulation; B- LAO view); Volume rendered images of heart showing absent LCX, LM continuing as LAD (C); posterolateral ventricular branch (PLV) arising from the RCA and extended leftwards, crossing the crux of the heart and then ascending into the inferior part of atrioventricular groove perfusing posterolateral and lateral walls of the heart (in the usual vascular territory of LCX artery; D)

dominant when it is extremely large and supplies the territory that is normally supplied by the other vessel [2]. In the largest retrospective survey of coronary anomalies as reported by Yamanaka and Hobbs et al [3], the incidence of anomalies of distribution was 1.15\%. Most of these cases have been reported in structurally normal heart, therefore they have been identified mostly as an incidental finding and rarely present with coronary artery disease.

Super-dominant RCA with absent LCA is a very rare anomaly with few reported cases in literature. This subtype of coronary anomalies is named type R-IA according to Lipton's classification and is by far the rarest type of single coronary artery, with an incidence of $0.0008 \%$ [4]. In this anomaly, the left main coronary artery continues as the LAD, while the RCA continues or gives rise to a distal branch that courses retrogradely to the left atrioventricular groove (i.e. the normal course of the left circumflex artery and coronary sinus) then to the left ventricle posterolateral wall (i.e. the LCA territory) [4].
Conventional angiography has been the gold standard for evaluating coronary artery anomalies (CAA) but availability of MDCT has made its evaluation easier, non-invasive and more fascinating because of excellent spatial resolution and rapid acquisition and therefore, it not only facilitates the visualization of these anomalies but also aids visualization of adjacent structure thus giving a fair idea of the potential outcome. Moreover, with the use of ECG gating, the movement and cardiac pulsation artefacts can be minimized. Magnetic resonance angiography (MRA) is another excellent modality in clearly identifying anatomy of anomalous coronary arteries but carries a limitation as it is not good for distal course of coronary arteries.

Absent left circumflex coronary artery with superdominant right coronary artery is a very rare anomaly where the left main coronary artery continues as left anterior descending artery and there is complete absence of the left circumflex artery and its branches. The right coronary artery is superdominant as its distal branches courses retrogradely in the left atrioventricular groove (in the course of the normal left circumflex artery) and supplies the left ventricle.

In one case study, the patient presented with symptoms of exertional angina [5]. It could have been due to transient ischemia of the left ventricular inferior and septal walls in conditions during which an increased oxygen demand is required. Normally, these areas are supplied by the left circumflex artery; however, in the absence of the left circumflex artery, the oxygen demand of these areas is supplied by the right coronary artery which may not be sufficient during physical exertion. So, the identification of this anomaly becomes important because the symptoms may mimic atherosclerotic coronary artery disease. In our case, exercise stress testing was terminated because of dyspnoea. It could have been angina equivalence or increased left ventricular end diastolic pressure because of underlying diastolic dysfunction and mild concentric left ventricular hypertrophy. Another possibility could have been syndrome $\mathrm{X}$ where patients present with chest pain, and angiogram reveals normal coronary arteries with no evidence of large- vessel involvement. 20\% of such patients may have positive exercise stress test. These patients often do not complete the exercise test because of fatigue or mild chest discomfort as in our case. Left ventricular function usually remains normal. Endothelial dysfunction, microvascular dysfunction and spasm are believed for responsible for angina in such patients. In some patients, there is abnormal pain perception, or sensitivity resulting into chest pain. Exaggerated preoccupation to personal health or panic disorder may be responsible in such cohort.

\section{Conflict of interest: None}

\section{References}

1. Angelini P (2007) Coronary artery anomalies: an entity in search of an identity Circulation 115:1296-1305. [Crossref]

2. Singh SP, Soto B, Nath H (1994) Anomalous origin of posterior descending artery from left anterior descending artery with unusual intraseptal course. $J$ Thorac Imaging 9: 255-277. [Crossref]

3. Yamanaka O, Hobbs RE (1990) Coronary artery anomalies in 126,595 patients undergoing coronary arteriography. Cathet Cardiovasc Diagn 21: 28-40. [Crossref]

4. Lin TC, Lee WS, Kong CW, Chan WL (2003) Congenital absence of the left circumflex coronary artery. Jpn Heart J 44: 1015-1020.

5. Alexander RW, Griffith GC (1956) Anomalies of the coronary arteries and their clinical significance. Circulation 14: 800-805. [Crossref]

Copyright: (C2021 Sinha S. This is an open-access article distributed under the terms of the Creative Commons Attribution License, which permits unrestricted use, distribution, and reproduction in any medium, provided the original author and source are credited. 